\title{
Educación de jóvenes y adultos (eja) desafíos de la diversidad en la realidad cotidiana del aula
}

\author{
Simone de Oliveira ${ }^{1}$ \\ Anderson Alves Santos ${ }^{2}$
}

Resumem. Desde hace años, se buscan métodos y prácticas educativas adecuadas a la realidad cultural y al nivel de especificidades de los jóvenes y adultos. La Educación de Jóvenes y Adultos (EJA) en la Red Municipal de Educación de Belo Horizonte - Minas Gerais, Brasil, prioriza la construcción de una política pedagógica para esta modalidad de enseñanza. El objetivo de esa investigación fue investigar, por medio de observaciones, por la percepción de los profesores, de los alumnos y la coordinación pedagógica, cuales son las principales estrategias pedagógicas desarrolladas para trabajar la diversidad entre los alumnos, característica importante en la EJA. El objeto de estudio fue una escuela de esta red de enseñanza que solamente trabaja con la modalidad EJA. La investigación describió y analizó, a partir da aplicación de encuestas a alumnos y profesores y de la observación directa de clases, las especificidades de los alumnos y el trabajo desarrollado por los profesores. En este orden de ideas, las metodologías utilizadas fueron el análisis bibliográfico y documental, el método histórico-lógico, el análisis y la síntesis. Una de las principales conclusiones es que existe un deseo manifiesto por parte de los profesores de trabajar las demandas de los alumnos y tener en cuenta sus especificidades, aún es necesario que los profesores continúen su formación para trabajar con la EJA y que la escuela priorice las discusiones, abordando en ellas el trabajo con la diversidad de los alumnos.

Descriptores: Educación. Enseñanza de Jóvenes y Adultos. Currículo.

\section{Education of Young people and adults (eja): challenges of diversity in the daily reality of the classroom}

\begin{abstract}
For years, it looks for educational methods and practices appropriate to the cultural reality and the level of the specific needs of young people and adults. The education of youths and adults (EJA) of the Belo Horizonte Municipal Education Network - Minas Gerais, Brazil, gives priority to the construction of an educational policy for this type of education. The objective of this research was to investigate, through observations, the perception of teachers, students and pedagogical coordination, which are the main pedagogical strategies developed to work the diversity among the students, an important characteristic in the EJA. The object of study was a school of this network of education that only works with the modality of EJA. The research described and analyzed, from the application of questionnaires to students and teachers and direct observation of the classes, the specific needs of the students and the work developed by the teachers. The methodologies used were the bibliographical and documentary analysis, the method of logico-logic, the analysis and synthesis. One of the main conclusions was that there is a manifest desire for teachers to work on the demands of the students taking into account their specificities, but it is still necessary for the teachers to continue their education to work with the EJA and that the school gives priority to the discussions, work with the diversity of students.
\end{abstract}

Keywords: Education. Youth and adult education. Curriculum.

\footnotetext{
${ }^{1}$ Mestre em Educação - Pedagoga da Secretaria Municipal de Educação de Betim. Psicopedagoga - Clínica Institucional - MG. solbhmg@yahoo.com.br

${ }^{2}$ Doutor e Professor do Instituto Federal de Minas Gerais - Câmpus - Formiga. MG. alvessanto@ gmail.com
} 
REVISTAELETRÔNICA DE GRADUAÇÃO

EPÓS-GRADUAÇÃO EMEDUCAÇĀO

REJ/UFG

\section{INTRODUCCIÓN}

A lo largo de la historia de la educación brasileña, la Educación de Jóvenes y Adultos - EJA siempre estuvo marcada por limitaciones y por la ausencia de políticas públicas específicas, en comparación con otras modalidades de enseñanza, estando por muchos años relegada a un segundo plano. Los avances han sido tenues y muchos problemas silenciados, dejando una deuda histórica con los jóvenes y adultos que no tuvieron ese derecho atendido en el Brasil, como por ejemplo, la escasez de inversiones en la formación y capacitación de profesores de la educación básica, lo que acentuó el distanciamiento de muchos posibles estudiantes de las escuelas.

De esa forma, fue innegable la contribución de Paulo Freire para la implantación en Brasil de una educación que tiene como foco principal el combate al analfabetismo, influenciando positivamente en los movimientos educacionales en nuestro país. Para Freire (2003, p.67), “[...] la educación es un proceso de humanización, de inserción y de transformación del mundo, que ocurre en diversos contextos sociales, entre los que se destaca la escuela." Es decir, por medio de la educación se ha hecho el proceso de inclusión social que promueve la formación, y, a partir de ésta, la mejoría de la cualidad de vida. Teniendo en cuenta que muchas personas no pudieron o tuvieron la oportunidad de dar continuidad a los estudios, la Enseñanza de Jóvenes y Adultos tiene como objetivo no sólo de suplir esa necesidad, sino de ampliar el horizonte de aquellos que la buscan.

La EJA como modalidad de enseñanza, tiene un compromiso pedagógico con los estudiantes, con su formación humana, con su autonomía, con el acceso al conocimiento universal, llevando al ejercicio de la ciudadanía, desarrollando su potencial crítico y reflexivo para que sean capaces de actuar y tener actitudes autónomas ante los desafíos cotidianos de la sociedad contemporánea. No obstante, se presenta en el actual contexto escolar una contradicción entre el currículo prescrito y el currículo aplicado en la realidad del aula, entre lo que se debe esperar y lo que realmente existe en este tipo de enseñanza. En la práctica, se presentan dos importantes cuestiones, que implican desafíos para los profesores: lograr desarrollar una propuesta pedagógica a partir de las Proposiciones Curriculares para la EJA que permita obtener mejores resultados en el trabajo desarrollado en clase, a través de una metodología que satisfaga las necesidades de todos los estudiantes y también trabajar en la práctica con la diversidad que caracteriza los grupos de EJA, diversidad que incluye ritmos 
diferenciados de aprendizaje, tiempo anterior de escolarización, edad, género, raza, credo, en fin, características muy marcadas y distintas.

\section{OBJETIVO}

El objetivo de esta investigación fue conocer algunas de las estrategias utilizadas por los profesores en las clases de la EJA Múltiples Edades y su efectividad, teniendo en cuenta la diversidad de los alumnos, haciendo un análisis de las perspectivas de los estudiantes, de los profesores y del coordinador pedagógico.

\section{MÉTODOS}

La metodología de la pesquisa consistió en la búsqueda y análisis de la bibliografía relacionada con el tema, incluyendo los instrumentos documentales que legislan la EJA, como en leyes, disposiciones y otros instrumentos legales, así como la bibliografía específica que fundamenta teóricamente la investigación, encontradas en muchas fuentes de información, como en libros, artículos, etc.

El método lógico histórico fue fundamental para definir la evolución histórica del problema, en este caso el desarrollo de la educación de jóvenes y adultos a lo largo de la historia de la educación de Brasil. Para Gamboa (2012), el método lógico histórico tiene como objetivo revelar determinaciones teóricas y presentar procesos de superación o transformación profesional y científico. El autor (2012, p.14) deja claro que este método se traduce en

[...] una herramienta de análisis epistemológico que permite, recuperar las características, o lógica interna o epistemología dominante en tesis y disertaciones y recuperar las condiciones materiales, institucionales y políticas que determinan dicha producción y revela sus perspectivas, inserción y tendencias dominantes en los campos profesionales y científicos, pero al mismo tiempo, ofrece para revelar los procesos de transformación y superación.

El método de análisis y síntesis, para Lakatos e Marconi (2000), es fundamental para que el desarrollo de la investigación tenga la ventaja de preparar al investigador para que sea capaz de seleccionar los diferentes elementos o partes de un fenómeno específico, y está relacionado con la capacidad sensorial de cada persona. En el proceso investigativo se observaron clases desarrolladas por los profesores de la escuela en grupos de Múltiples Edades. Ese procedimiento metodológico se tomó en función de que este permite 
que el observador llegue más cerca de la "perspectiva de los sujetos", un importante blanco de los abordajes cualitativos. En la medida en que el observador acompaña in loco las experiencias diarias de los sujetos, puede tratar de comprender su visión del mundo. Esto es, el significado que ellos atribuyen a la realidad que los rodea y a sus propis acciones (LÜDKE \& ANDRÉ, 1986, p. 26).

\subsection{Objeto de estudio}

La escuela estudiada hace parte de la red municipal de educación de Belo Horizonte, ofreciendo clases de enseñanza fundamental y media exclusivamente en la modalidad de EJA. Desde 1991, la escuela ofrece EJA en las tres sesiones de trabajo: mañana, tarde y noche, lo que garantiza opciones de estudios para todos los interesados, según sus posibilidades de horarios.

Los estudiantes que sirvieron de base del análisis son parte de la clase mixta (múltiples edades); muchos están en proceso de la alfabetización y otros en el proceso de certificación. Setenta cuestionarios fueron distribuidos para los estudiantes y sirvieron de base del análisis. El perfil de los estudiantes analizados son jóvenes que no adaptaron a la enseñanza regular; adultos y ancianos que tienen diversas experiencias y niveles de aprendizaje. También han hecho parte de lo objeto de estudio ocho profesores que trabajan con los grupos y el coordinador que hace el acompañamiento pedagógico. Los profesores fueron aprobados por concurso público para el trabajo en la alcaldía de Belo Horizonte, pero no específicamente en la educación de jóvenes y adultos. El perfil de los docentes es muy distinto, porque son de diferentes áreas del conocimiento. Ellos están más de 8 años en la educación, pero no han recibido formación específica para trabajar con esta modalidad de enseñanza.

Para la coleta de dados fueron utilizados y aplicados cuestionarios semiestructurados, que de acuerdo con Gil (1999), cuestionario es la técnica de investigación que tiene como objetivo el conocimiento de opiniones, creencias, interés, expectativas, situaciones vivenciadas, para los alumnos - que tuvieran como objetivo de conocer sus características personales para detectar hasta dónde existe diversidad entre ellos; y para los profesores, para conocer acerca dos métodos utilizados y las dificultades que se presentan en el proceso de enseñanza-aprendizaje. También se elaboró un cuestionario para el coordinador pedagógico de la escuela, quien representa a la Secretaría Municipal de Educación, para conocer sus opiniones acerca del trabajo general de la EJA y el de la escuela. 
Independientemente de que mucho se ha escrito al respecto, existen aspectos poco tratados, al menos en la práctica, como es el caso del tratamiento a la diversidad en las clases por parte de los profesores. De hecho, tratar este problema en una escuela que solamente se dedica a esta modalidad de enseñanza puede aportar ideas en cuanto a que hacer para trabajar las diferencias, la heterogeneidad, que se presenta en los estudiantes, lo que es de gran importancia para los resultados que se pueden obtener.

\section{FUNDAMENTACIÓN TEÓRICA}

Cuando se trabaja en el día a día del aula, es necesario reflexionar acerca de qué enseñar y cómo enseñar. Un currículo para la Educación de Jóvenes y Adultos exige una visión dirigida a comprender no solo lo que ya fue desarrollado por esa modalidad de enseñanza, sino también cómo establecer una nueva percepción de lo que representa la EJA, más dirigida al desarrollo de la ciudadanía, de acuerdo con los intereses y necesidades de los educandos. Sacristán (2000, p. 125) destaca que

[...] el término currículo viene de la palabra latina curriculum, cuya raíz es la misma de currere, que se refiere a la carrera, al camino a ser andado. El tratamiento del currículo actualmente presupone que se observe su problemática a partir de la reflexión acerca de: qué objetivo se pretende alcanzar, qué enseñar, por qué enseñar, a quién están dirigidos los objetivos, quién posee mejor acceso a las formas legítimas de conocimiento, qué procesos inciden y modifican las decisiones hasta que se llegue a la práctica, cómo se transmite la cultura escolar, cómo los contenidos se pueden interrelacionar, con qué recursos/materiales metodológicos, cómo organizar los grupos de trabajo, el tiempo y el espacio $[\ldots]$

A partir de la reflexión sobre la práctica, como plantea Sacristán, el profesor puede buscar, en el contexto en el que está insertada su realidad, herramientas que lo auxilien en la elaboración de estrategias de enseñanza que respondan mejor a las especificidades de los alumnos. Los objetivos que se pretenden alcanzar, permitiendo el acceso al conocimiento de estos sujetos, mantienen el compromiso con la formación humana y el ejercicio de la autonomía. Este mismo autor aborda aspectos del currículo, de cómo el profesor puede elaborar una propuesta pedagógica que esté en consonancia con una educación justa y de calidad, y cómo cambiar las prácticas escolares de forma tal que puedan atender a los jóvenes y adultos que entran en esta modalidad de enseñanza. En esa perspectiva, César Coll, en "Psicología y Currículo" (1987, p. 45) también afirma que 
[...] el currículo preside a las actividades educativas escolares, define sus intenciones y proporciona las guías de acción adecuadas y útiles para los profesores, que son directamente responsables por su ejecución.

Coll destaca la importancia de definir las intenciones educativas adecuadas. No obstante el engranaje que se encuentra estructurado al "quehacer pedagógico" demuestra nuestra inquietud cuando no cuestionamos la propuesta curricular a la que sometemos a nuestros alumnos. El gran desafío en trabajar con las dificultades presentadas por los alumnos, priorizando su cultura, su historia de vida, sin dejar de lado las directrices curriculares, está presente en las discusiones entre los profesores.

\section{Márquez enfatiza en su artículo "Factores Asociados al Fracaso Escolar en la}

Educación Secundaria de Huelva", que la escuela puede hacer diferencia para el éxito de los estudiantes a través de la didáctica, de el currículo, de los métodos de enseñanza y de las relaciones personales:

La organización escolar juega un papel decisivo en el desarrollo educativo de sus miembros. A través de ella puede concretarse la construcción de una escuela inclusiva y reductora de desigualdades (González, 2008; Lorente, 2006; Muñoz, Rodríguez y Barrera, 2013). Los modelos de dirección educativa, la formación del profesorado, los métodos de enseñanza-aprendizaje o las relaciones personales que se establecen en el interior de las aulas contribuyen a que los procesos educativos sean ricos y valiosos para todos (Baerveldt, Van de Bunt y de Federico, 2010; González, 2002; McMahon, 2009; Murillo y Hernández-Castilla, 2011). De esta forma, la escuela cumpliría su función social a través de un currículo y una organización escolar al servicio de la didáctica pero lamentablemente, esta no parece ser la realidad de un sistema educativo que, como el nuestro, no garantiza un contexto motivador de aprendizaje que minimice los riesgos de fracaso escolar (BIRBILI, 2005; CALERO, 2006).

En relación a los currículos, Torres (2011) analizó proyectos de elaboración de políticas de currículo para la EJA en dos espacios que circulan diferentes textos que presentan demandas de diversos grupos: los Encuentros Nacionales de Educación de Jóvenes y Adultos (ENEJAs) y el GT 18 de la Asociación Nacional de Posgrados e Investigación en Educación (ANPEd). Se identificaron y problematizaron demandas en disputa en cuanto a la elaboración de políticas de currículo en la EJA que, articuladas al proceso de significación, tienen la intención de construir un discurso hegemónico en el currículo de la EJA.

En el proceso de producción de las políticas, se destacó la actuación de comunidades epistémicas en diferentes contextos, en la tentativa de influenciar y hegemonizar 
determinados sentidos en cuanto a la producción de las políticas de currículo en la EJA. En este sentido, las políticas de currículo se consideraron como discurso, lo que implicaba abordar y problematizar discursos que aparecen en diferentes contextos entrelazados por relaciones de saber - poder.

Para ello, el autor "dialogó" con la teoría del discurso propuesta por Ernesto Laclau, el enfoque metodológico del ciclo continuo de políticas de Stephen Ball y la vertiente analítica de las comunidades epistémicas, argumentando que las políticas de currículo se producen en diferentes contextos, con el envolvimiento de diferentes actores sociales. Basándose en el análisis de diversos documentos y demandas, defendió qué posibles discursos se constituyen en función de la articulación de ciertas demandas equivalentes que procuran hegemonizar determinados sentidos de/en la política curricular de la EJA.

Así, la realidad pedagógica vivida en esta modalidad de enseñanza, me motivó a investigar acerca de las posibilidades reales en la relación existente entre el currículo y las estrategias y prácticas pedagógicas que desarrollan los profesores de la EJA en una escuela en Belo Horizonte, que invierte en este tipo de enseñanza con el objetivo de proporcionar a los jóvenes, adultos y ancianos que fueron excluidos de la escolarización formal, la oportunidad de disfrutar de su derecho fundamental a la educación.

En 2013, hubo en la ciudad de Belo Horizonte, un debate curricular, con la participación de asesores, consultores y colaboradores, que culminó con la publicación en 2014 de la versión preliminar del documento con las Propuestas Curriculares para la EJA en el municipio. Este documento es un orientador de la práctica para nuevas acciones y reflexiones sobre la EJA.

En la pesquisa documental fue posible conocer la organización de la EJA en la Red Municipal de Educación de Belo Horizonte, por medio de la Resolución 317/2014/SMED (Secretaria Municipal de Educación, de la ciudad de Belo Horizonte/Minas Gerais), que establece dos modalidades de la Educación de Jóvenes y Adultos, EJA Múltiples Edades y EJA Juvenil. Es importante dejar claro que las resoluciones son actos administrativos normativos que salen de una autoridad superior, por el que regulan las materias de su competencia específica. Las resoluciones no pueden contradecir las normas y reglamentos, pero explicarlos. La EJA Múltiples edades, objeto de esta investigación, tiene una atención en ciclo único de 
formación, con una duración máxima de 1920 horas, teniendo como público, estudiantes mayores de 15 años, alfabetizados o no, que no concluyeron la enseñanza fundamental. Mientras la EJA Juvenil se plantea una atención al alumno con duración anual de 600 horas, para estudiantes alfabetizados de edades entre 15 y 18 años, con discrepancia de escolaridad de por lo menos dos años.

\section{RESULTADOS Y DISCUSIONES}

\subsection{Análisis desde la óptica de los educadores}

Los datos colectados a partir de la aplicación de la encuesta posibilitaron algunas análisis importantes. En relación con las diferencias fundamentales entre las dos formas de organización de la EJA, los encuestados logran comprender las propuestas y señalan la docencia única como el desafío principal para los profesores, además de la diversidad del público con el que trabajan.

La Propuesta Político Pedagógica es el principio orientador para la escuela. Todos los docentes afirman conocer la propuesta, señalando algunos puntos relevantes, tales como: valorización de los saberes adquiridos por los estudiantes; utilización de los espacios educativos de la ciudad; énfasis en la lectura; interpretación de diferentes géneros textuales; inclusión de alumnos con deficiencias; calificación profesional; organización de los conocimientos; y la diversidad. Con relación a las Proposiciones Curriculares para la EJA, aún acontecen discusiones y lecturas del documento para la planificación del quehacer pedagógico.

En cuanto a la caracterización de los estudiantes, aspecto fundamental para esta investigación, los docentes destacaron como aspecto fundamental la diversidad existente: diversos intereses personales y profesionales; distintos saberes culturales, experiencias de vida; intereses para la continuidad de estudios y ascenso profesional; diferentes edades, profesiones y ocupaciones; ritmos diferenciados de aprendizaje (los de más edad son más lentos), diferencias de intereses (los más jóvenes son menos interesados); estudiantes con dificultades cognitivas, visuales y auditivas; alumnos hiperactivos. En fin, una heterogeneidad que requiere un trabajo individualizado y cuidadoso para atender a ese público.

En relación con los proyectos desarrollados por la escuela, los profesores mencionan los talleres de artes, teatro, "Frutos de la EJA" (proyecto en el que los alumnos 
fabrican materiales de limpieza), talleres de producción de cosméticos, con orientación de los profesores. También hacen referencia al proyecto sobre el Mundo del Trabajo, entre otras actividades. Existen también visitas a espacios culturales de la ciudad, en las que se trabaja la ciudadanía y la historia; clases de contadores de cuentos; proyectos de lectura; práctica de Yoga y muchas actividades que se desarrollan con el objetivo de atender a los estudiantes en cuanto a sus dificultades cognitivas.

En las clases, los profesores afirman que se auxilian de actividades colectivas, vídeos y todos los recursos didácticos disponibles. Utilizan la biblioteca, desarrollando actividades diferenciadas para cada nivel de aprendizaje, usando materiales elaborados en letras mayúsculas con los alumnos que están en proceso inicial de alfabetización, folletos de propaganda de supermercados, farmacias y atención individualizada, como estrategias utilizadas para la atención a ese público, teniendo en cuenta sus especificidades.

Apuntan sugerencias y mejorías, teniendo en cuenta la diversidad de los alumnos: la realización de más acuerdos con instituciones que ofrecen cursos profesionales en diversas áreas, para dar continuidad a la formación de los profesores que actúan en la EJA, haciendo énfasis en la práctica; orientación especializada para los alumnos con problemas más graves; alianzas con instituciones que enfatizan el mundo del trabajo; convenios con universidades. En ese sentido, es importante dar voz al relato de los profesores. Numerosos estúdios muestran la importância del profesor en el proceso educativo. Cervini, Dari, Quiroz y Atorresi $(2013$, p. 1) afirman que

En gran medida, dentro del aula acontece la enseñanza-aprendizaje, un proceso multidimensional en el que las características del maestro y lo que él hace constituyen aspectos claves. Por ello, una gran parte de la investigación sobre eficacia escolar se ha esforzado por identificar las diversas características del proceso de enseñanza en el aula que tienen efectos sobre el aprendizaje de los alumnos.

\subsection{Análisis desde la óptica de los estudiantes}

Sobre la base de la encuesta aplicada a los alumnos, los datos demuestran que hay un porciento mayor de mujeres y que el grupo con edades mayores a 40 años también es el mayor, lo que significa que una cantidad expresiva de alumnos que quieren comenzar o regresar a estudiar se encuentra en la fase adulta avanzada, incluyendo la tercera edad. 
En cuanto al origen étnico de los alumnos, la mayoría son pardos y negros, pero algunos optaron por no responder a esta pregunta; o sea, que no logran o no quieren se encuadrar en ninguna de las opciones propuestas. Esta situación puede ser interesante, ya que el no encuadramiento étnico racial puede significar hasta temor al racismo, lo que puede ser un indicador importante que motive trabajar este aspecto social en las clases como uno de los elementos de diversidad en la población del Brasil y que estudia en la EJA.

Analizando el origen de los estudiantes en cuanto a la ubicación de su hogar en relación a la escuela, la mayoría afirma que viven en barrios distantes de la escuela (lo que demuestra su interés en estudiar y recuperar el tiempo fuera de la escuela).

Observando los datos obtenidos a partir de las encuestas, se puede percibir que la mayoría de los que estudian en la EJA son trabajadores y que muchos están tratando de recuperar el tiempo perdido y que quieren adquirir un diploma para mejorar en su vida. En cuanto a la escolaridad antes de entrar a la EJA, la mayoría afirma nunca haber estudiado en otras modalidades de enseñanza. Esto significa que la mayoría de los que comienzan a estudiar en la EJA deben ser prácticamente analfabetos o analfabetos funcionales, ya que nunca estudiaron. También se les preguntó sobre los motivos para procurar la EJA.

Los alumnos afirmaron también lo que más les llama la atención en la EJA es el metodología utilizada, lo que está estrechamente relacionado con otras respuestas importantes, como las características de los profesores y la forma como son acogidos por ellos. Destacan que pretenden continuar sus estudios después de obtener el certificado. Este resultado es importante, porque de forma directa refleja el interés de los alumnos por los estudios y lo que ellos pueden significar en sus vidas. Los objetivos para la continuación de los estudios fueron, en primer lugar, poder progresar en su vida y conseguir un trabajo mejor, seguido del interés de tener un certificado, lo que, de hecho, está muy relacionado con el primero de ellos, ya que obtener un certificado puede ser importante para nuevas posibilidades de desarrollo, tanto personal como profesional y laboral.

Estos últimos aspectos reflejan claramente algunas características de los alumnos que frecuentan la EJA, destacándose el hecho que el interés y expectativas por mejorías en la actividad laboral representar un factor más que decisivo para su incorporación a los estudios. 
REVISTAELETRÔNICA DE GRADUAÇÃO

EPÓS-GRADUAÇÃO EMEDUCAÇĀO

REJ/UFG

\subsection{La óptica del acompañamiento pedagógico}

La actividad de acompañamiento pedagógico existe en las escuelas del municipio como una forma de conocer el que hacer pedagógico de cada centro de estudios, dando el soporte necesario a todas las actividades desarrolladas en ellas. En cuanto a los desafíos de su trabajo, el profesional entrevistado, señala el hecho de tener que conciliar la política de la Secretaria de Educación con los intereses específicos de la escuela. Sobre el hecho de existir dos formas de organización de la EJA en Belo Horizonte se debe a las diferencias de edades, diferentes expectativas en cuanto al futuro y comportamiento, entre otras causas.

El joven y el adolescente, de forma general, esperan dar continuidad a los estudios para tener una profesión. El adulto espera mejores posibilidades en el trabajo, o de trabajo. Por su parte, los de mayor edad esperan poder leer algo, como por ejemplo, la biblia o un periódico. También pretende tener momentos de convivencia social con sus parejas y poder vencer una etapa que no pudo concluir en su juventud. La organización de las EJA Ju venil tiene entonces que preocuparse en ser rápida, eficiente, atractiva y debe cumplir el papel de capacitar para la continuidad de estudios. La EJA debe preocuparse en propiciar el acceso a la alfabetización, a los conocimientos básicos y posibilitar la continuación de estudios futuros, pero sin presión de tiempo. Para ello, debe ser flexible en cuanto a su organización y reconocer los conocimientos adquiridos anteriormente.

En cuanto al perfil de sus alumnos, hace énfasis en el hecho de que la escuela, por trabajar solamente con la EJA, recibe alumnos de todos los tipos, con expectativas, escolaridad y comportamientos diferentes. En cuanto a los grupos de edades, la escuela tiene adolescentes, jóvenes, adultos y ancianos, predominando los adolescentes como consecuencia de la creación de los grupos de EJA Juvenil. Se evalúa como positiva la característica que marca la escuela: una posición cuestionadora. Aunque se considera que aún no desarrolló una práctica que tenga en la diversidad un aspecto fundamental, existe una búsqueda para entender mejor cómo actuar en esta situación. Además, es importante valorizar la persistencia de los educadores en cuanto a hacer de la escuela una institución con foco en el mundo del trabajo. Señala que existen aspectos que son importantes seguir siendo discutidos. Considera que una alteración de la Propuesta Política Pedagógica (PPP) de la escuela podría ser interesante, porque promovería 
discusiones acerca de la propia concepción de la EJA. Finalmente, teniendo en cuenta la diversidad, según él, la escuela ofrece campo muy fértil para el diálogo.

\section{CONCLUSIONES}

En este proceso de investigación se pudo verificar la gran dificultad enfrentada por los educadores de la EJA que, muchas veces, no fueron preparados para dar cuenta de esta diversidad; pero realizan un gran esfuerzo y que, aún con las dificultades diarias, tratan de diversificar sus estrategias didácticas. Percibe entonces que es necesaria una formación continua para estos docentes que se proponen trabajar con este público. Y más allá de la formación, está el deseo, la mirada diferenciada, para que el educador pueda lidiar con la diversidad que presenta este público.

Un hecho muy interesante es la forma como los profesores interactúan con los alumnos, procurando establecer relaciones interactivas e influyendo positivamente en la vida de sus estudiantes, proporcionando una influencia directa en el aprendizaje. Es una escuela que ofrece muchas posibilidades para los estudiantes por medio de las conferencias, talleres de arte, excursiones y actividades distintas que hacen diferencia para el aprendizaje de los alumnos. Los profesores se cuestionan pedagógicamente y cuestionan a sus alumnos para saber en qué medida ellos se sienten o no atendidos en cuanto a sus necesidades.Y allá de las decisiones políticas de las instancias gestoras, el sujeto principal, mediador del conocimiento es el profesor, así es fundamental tener en cuenta sus sugestiones para mejorar el trabajo.

En la observación del trabajo en el aula, de la rutina de las clases, de la práctica pedagógica y específicamente de la práctica docente, fue posible percibir que la concepción de los profesores sobre el currículo de la EJA ya no estaba limitado al paradigma tradicional, pues ya trabajaban con algunas perspectivas emancipadoras. Esto se manifestaba en el diálogo, en las actividades, en las relaciones interactivas.

En este sentido, el currículo, muchas veces, desarrollado a partir de las estrategias y recursos didácticos disponibles, como libros, revistas, internet, trabajos en grupo e intercambio de experiencias, presenta avances en medio a los desafíos manifestados por los profesores. Así mismo, ante tales dificultades, los estudiantes afirman que la metodología es uno de los aspectos que más les llama la atención en la EJA, se crea entonces una expectativa con respecto a la 
atención de las demandas de estos alumnos. Es importante enfatizar que en el proceso de enseñanza-aprendizaje, propuestas flexibles y bien planificadas puedan atender de forma más eficiente las especificidades de este grupo heterogéneo y posibilitar su inclusión en el mundo del conocimiento. Paulo Freire, que contribuyó fuertemente con la discusión curricular para la EJA, y en su trayectoria, defendió la enseñanza como forma de despertar el sentido crítico del alumno, haciendo que el mismo buscase ampliar su conciencia social y lograr alcanzar la autonomía ante su realidad de vida, afirma que "en mundos diferentes la educación es diferente" (FREIRE, 1984, p.39).

Eso lleva a creer que no hay una mejor solución para que el profesor trabaje la diversidad, ya que no existe una única propuesta que pueda dar respuesta a todas las especificidades de la EJA, sino posibilitar que los alumnos logren contextualizar históricamente sus experiencias de vida, relacionándolas, junto con sus conocimientos con los aspectos estudiados, que puedan interactuar con sus colegas, participar en proyectos que contribuyan para un aprendizaje significativo. Este puede ser, sin dudas, uno de los caminos hacia el logro de buenos resultados en la educación de estos jóvenes y adultos.

Cuando se refiere a la universalización del saber, y para que ese derecho sea efectivo, es necesario garantizar no solamente el acceso a la escuela, sino también la permanencia del alumno en ella. Esto significa que la atención tiene que ser más calificada, teniendo en cuenta la diversidad en cuanto a clase social (áreas de la periferia, pobreza, desigualdad y vulnerabilidad social), las relaciones entre personas de diferentes edades y generaciones, las diferencias de género y orientación sexual, de razas y etnias y de religión. En este sentido, Arroyo (2006, p. 24) afirma que "es importante trabajar con proyectos que tengan en cuenta su realidad y su condición. Sabemos muy poco sobre el desarrollo de esa juventud, de esos jóvenes y adultos populares, con trayectorias humanas cada vez más precarias".

Otro aspecto que se debe tener en cuenta es la distancia geográfica de muchos estudiantes en relación con la escuela en la que estudian. Debe trabajarse para lograr ofrecer esta modalidad de estudios en una red más amplia de escuelas, de forma tal que los alumnos tengan que dislocarse lo menos posible para asistir a clases. Como se pudo observar en las encuestas aplicadas, un número significativo de estudiantes viven en barrios distantes de la escuela en la que estudian, lo que puede resultar en un elemento que dificulte su permanencia en el centro de estudios. Si hubiera más oferta de escuelas con la modalidad EJA, 
REVISTAELETRÔNICA DE GRADUAÇÃO

EPÓS-GRADUAÇÃO EMEDUCAÇĀO

REJ/UFG
REFLETIONIS

NÜMERO ESPECIAL VoL. 15 N ${ }^{-2} 212019$

probablemente no solo se garantizaría la permanencia de los que hoy estudian, sino que se lograría una mayor incorporación de estudiantes en la EJA.

En relación al perfil de los estudiantes, la mayoría de ellos son pardos y negros (64\%) y mujeres (62\%), que históricamente ya fueron excluidos del sistema educacional. Se pudo deducir que los aspectos étnico raciales y de género son indicadores que necesitan ser evaluados como elementos de reflexión al pensarse en un currículo para la EJA, de forma tal que no se reproduzcan los patrones ya establecidos por una sociedad blanca y machista, que permite que los grupos dominantes perpetúen sus paradigmas. Ya no es posible trabajar en la educación con la idea de excluir al "diferente". En una educación de calidad se tienen que incluir a todos, teniendo siempre en cuenta las especificidades y anhelos de los alumnos.

Es necesario percibir a estos estudiantes de forma diferenciada, con la sensibilidad necesaria que permita comprender sus necesidades, sus ritmos de aprendizaje, entender que la mayoría de ellos son trabajadores que quieren mejorar su nivel de escolaridad para progresar en el mundo laboral, o hasta conseguir un empleo que le permita sobrevivir. No existe una forma o modelo únicos de atención en la EJA que dé respuesta a todas las especificidades de sus alumnos. Mientras existe un grupo que demanda de una atención presencial, hay otros que necesitan de una organización más flexible. El desafío del poder público es entonces ofrecer varios modelos de atención y no uno solo.

Es importante destacar también que, la relación que los jóvenes y adultos de diferentes edades establecen con el conocimiento, depende de la forma como la institución los acoge. Cuando los educadores logran extrapolar la cuestión del contenido que va a ser trabajado y se establece un diálogo más "afectuoso" y una relación intermediada por situaciones interactivas, hay un significativo aumento de la motivación y, consecuentemente, una mayor permanencia de los estudiantes en la escuela. Esta escuela busca estrechar las relaciones interpersonales entre alumnos y profesores, siempre garantizando el respeto mutuo a fin de garantizar una mejor acogida de los alumnos en el centro de estudios, contribuyendo significativamente al logro de mejores resultados por parte de los alumnos. Este vínculo proporciona una mejor posibilidad de aproximación del sujeto en relación con el conocimiento, porque después de la metodología, lo que más llama la atención de los alumnos de la EJA son sus profesores. 
Por tanto, es imprescindible tener en cuenta el perfil del profesor que trabaja en esta modalidad de estudios. Saber si este profesor desea realmente trabajar en la EJA, si está en sintonía con las expectativas de sus alumnos y si está dispuesto a participar de una formación continua que lo capacite cada vez más para desarrollar un trabajo de calidad, ampliando sus horizontes en las prácticas educativas. Para trabajar con la educación, especialmente para desarrollar una escolarización humanizada y posible para jóvenes y adultos, el profesor, protagonista y mediador del conocimiento, tiene que entender que lidiar con la pluralidad de sujetos es un factor importante para tener éxito en su trabajo. Y aún cuando no haya cursado ninguna disciplina específica sobre EJA en su carrera, lo que de hecho es un problema en un país en el que existe un número significativo de personas no alfabetizadas, debe entender que la formación continua es el camino para la mejoría de sus prácticas en este tipo de enseñanza. En este sentido, el deseo, la disposición para aprender a enseñar, debe ser el elemento impulsor de quien trabaja en la EJA. El profesor solo podrá enseñar si está dispuesto a aprender.

\section{REFERENCIAS}

ARROYO, Miguel. Formar educadoras e educadores de Jovens e Adultos. IN: SOARES, Leôncio (org.). Formação de educadores de Jovens e Adultos. Belo Horizonte: Autêntica/SECAD-MEC/UNESCO, 2006.

BELO HORIZONTE. (2013). Secretaria Municipal de Educação. Versão preliminar das Proposições Curriculares para a EJA. SMED.

BELO HORIZONTE. (2014). Secretaria Municipal de Educação de Belo Horizonte - SMED. PORTARIA SMED N 317/2014 Dispõe sobre a organização para o Ensino Fundamental Regular, para a modalidade Educação de Jovens e Adultos - EJA,

CERVINI, R.; DARI, N.; QUIROZ, S.; ATORRESI, A. (2014). Maestro, aula y aprendizaje en América Latina. Los datos del SERCE. REICE. Revista Iberoamericana sobre Calidad, Eficacia y Cambio en Educación, 12 (2), 105-137. Disponible en: http://www.rinace.net/ reice/numeros/vol12num2.htm. Acesso en: 28 jun. 2017.

COLL, Cesar. (1987). Psicologia e Currículo. São Paulo: Ática.

FREIRE, Paulo. (1984). Educação como prática da liberdade. Rio de Janeiro: Paz e Terra.

FREIRE, Paulo. (1984). Pedagogia do oprimido. Rio de Janeiro: Paz e Terra.

FREIRE, Paulo. (2003). Pedagogia da autonomia: saberes necessários à prática educativa. São Paulo: Paz e Terra.

GAMBOA, S. S. Pesquisa em Educação: métodos e epistemologias. Chapecó, SC: Argos, 2012. In: Gamboa, Sílvio Sánchez; Gamboa, Márcia Chaves. O método lógico-histórico nas 
análises epistemológicas: a experiência brasileira no campo da educação física Filosofia e Educação (Online). Volume 6, Número 2, Junho de 2014. Disponible en:

http://ojs.fe.unicamp.br/ged/rfe/ article/view/6388/5319. Acesso en: 17 jul. 2017.

GIL, Antônio Carlos. (1999). Métodos e técnicas de pesquisa social. $5^{\text {a }}$ ed. São Paulo: Atlas.

LAKATOS, E. M.; MARCONI, M. A. (2000). Fundamentos de metodologia científica. 6. ed. São Paulo: Atlas.

LÜDKE, M.; ANDRÉ, M. E. D. A. Pesquisa em educação: abordagens qualitativas. São Paulo: EPU, 1986.

MÁRQUEZ, C. (2016). Factores asociados al fracaso escolar en la educación secundaria de Huelva. REICE. Revista Iberoamericana sobre Calidad, Eficacia y Cambio em

Educación, 14 (3), 131-144. Disponible en: http://www.rinace.net/reice/numeros/arts/vol14 num3/art7.htm. Acesso en: 12 set. 2017

SACRISTÁN, J. Gimeno. (2000). Compreender e transformar o ensino. Porto Alegre: Artmed.

TORRES, Wagner Nóbrega. (2011). Políticas de currículo em educação de jovens e adultos (EJA). Dissertação (mestrado) - Universidade do Estado do Rio de Janeiro. Faculdade de Educação. Disponible en: http://www.bdtd.uerj.br/tde_busca/ arquivo.php? codArquivo=5383 Acesso en: 20 set. 2017. 